\title{
GCN5 is a required cofactor for a ubiquitin ligase that targets NF- $\mathrm{B} / \mathrm{RelA}$
}

\author{
Xicheng Mao, ${ }^{1,2}$ Nathan Gluck, ${ }^{1,2}$ Duo Li, ${ }^{3}$ Gabriel N. Maine, ${ }^{1,2,4}$ Haiying Li, ${ }^{1,2}$ Iram W. Zaidi, ${ }^{1,2}$ \\ Aparna Repaka, ${ }^{1,2}$ Marty W. Mayo, ${ }^{3}$ and Ezra Burstein ${ }^{1,2,5,6,7}$ \\ ${ }^{1}$ Department of Internal Medicine, University of Michigan Medical School, Ann Arbor, Michigan 48109, USA; ${ }^{2}$ Molecular \\ Mechanisms of Disease Program, University of Michigan Medical School, Ann Arbor, Michigan 48109, USA; ${ }^{3}$ Department of \\ Biochemistry and Molecular Genetics, University of Virginia, Charlottesville, Virginia 22908, USA; ${ }^{4}$ Department of Clinical \\ Pathology, William Beaumont Hospital, Royal Oak, Michigan 48073, USA; ${ }^{5}$ Gastroenterology Section at the Ann Arbor VA \\ Medical Center, Ann Arbor, Michigan 48105, USA
}

The transcription factor NF-кB is a critical regulator of inflammatory and cell survival signals. Proteasomal

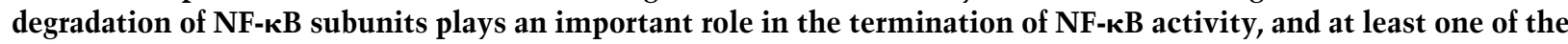
identified ubiquitin ligases is a multimeric complex containing Copper Metabolism Murr1 Domain 1 (COMMD1) and Cul2. We report here that GCN5, a histone acetyltransferase, associates with COMMD1 and other components of the ligase, promotes RelA ubiquitination, and represses $\kappa \mathrm{B}$-dependent transcription. In this role, the acetyltransferase activity of GCN5 is not required. Interestingly, GCN5 binds more avidly to RelA after phosphorylation on Ser 468, an event that is dependent on IKK activity. Consistent with this, we find that both GCN5 and the IKB Kinase (IKK) complex promote RelA degradation. Collectively, the data indicate that GCN5 participates in the ubiquitination process as an accessory factor for a ubiquitin ligase, where it provides a novel link between phosphorylation and ubiquitination.

[Keywords: NF-кB; RelA; GCN5; COMMD1; ubiquitin; Cul2]

Supplemental material is available at http://www.genesdev.org.

Received October 3, 2008; revised version accepted February 25, 2009.

NF-кB is a dimeric transcription factor formed by members of a highly conserved family of proteins that share an 300-amino-acid region termed the Rel Homology Domain (RHD) (Karin and Lin 2002). The activity of NF-кB is primarily regulated by cytoplasmic sequestration of the NF-кB dimer through its interaction with inhibitory ІкB proteins (Baeuerle and Baltimore 1988). Activation of the multimeric IкB Kinase (IKK) results in the phosphorylation of IкB, followed by its ubiquitination and degradation (Henkel et al. 1993; Chen et al. 1995), allowing the translocation of NF-kB into the nucleus. Nuclear NF-kB dimers bind to an array of promoters, ultimately resulting in the induction of genes involved in processes such as immunity, apoptosis, cell cycle progression, and oncogenesis (Silverman and Maniatis 2001). Termination of NF$\kappa \mathrm{B}$ activity is mediated by resynthesis of I $\mathrm{B}$ proteins, which facilitate nuclear export of NF-кB (Arenzana-Seisdedos et al. 1997). More recently, additional mechanisms for

${ }^{6}$ Present address: Department of Internal Medicine, University of Texas Southwestern Medical Center at Dallas, 5323 Harry Hines Blvd., Room J5.126, Dallas, TX 75390-9151, USA.

${ }^{7}$ Corresponding author.

E-MAIL ezra.burstein@utsouthwestern.edu; FAX (214) 648-2022.

Article is online at http://www.genesdev.org/cgi/doi/10.1101/gad.1748409. transcriptional regulation have been recognized, including post-translational modifications of NF- $\mathrm{B}$ subunits such as phosphorylation (Zhong et al. 1998; Sakurai et al. 1999), acetylation (Chen et al. 2001; Kiernan et al. 2003), prolyl-isomerization (Ryo et al. 2003), and ubiquitination (Saccani et al. 2004; Maine et al. 2007; Tanaka et al. 2007).

Copper Metabolism Murr1 Domain-containing (COMMD) proteins are a group of evolutionarily conserved factors present in a wide range of organisms (Burstein et al. 2005). COMMD1, the prototype member of the family, binds to a conserved domain present in the RHD of all NF-кB subunits (Ganesh et al. 2003; Burstein et al. 2005) and inhibits NF-kB-mediated transcription, leading to decreased proinflammatory gene expression (Maine et al. 2007) and impaired HIV-1 viral replication (Ganesh et al. 2003). We recently reported that COMMD1 inhibits NF-кB by promoting the ubiquitination and proteasomal degradation of NF-кB subunits (Maine et al. 2007). COMMD1 interacts with a multimeric ubiquitin ligase that also contains Elongins B and C, Cul2, and SOCS1 that is capable of ubiquitinating NF-кB subunits (Ryo et al. 2003; Maine et al. 2007). Interestingly, another report implicates IKK $\alpha$ mediated phosphorylation of RelA to the subsequent degradation of this NF- $\kappa$ B subunit, although the precise 
pathway of degradation has not been elucidated (Lawrence et al. 2005). In particular, any potential links between COMMD1-mediated ubiquitination and the phosphorylation of RelA are not known.

Here we report that GCN5, a histone acetyltransferase (HAT), is a COMMD1-associated factor. Unlike its wellknown role as a transcriptional activator (Thomas and Chiang 2006), we find that GCN5 functions as a repressor of NF- $\mathrm{KB}$ by promoting the ubiquitination and degradation of RelA, an activity consistent with its interaction with the COMMD1-containing ligase. Interestingly, GCN5 binds primarily to the $\mathrm{C}$ terminus of RelA, and this interaction is enhanced by IKK-dependent phosphorylation on Ser 468. The ability of GCN5 to bind to phosphorylated RelA and to the COMMD1 complex provides a link between IKK-mediated phosphorylation, ubiquitination, and the termination of $\kappa \mathrm{B}-$ mediated transcription.

\section{Results}

\section{Identification of GCN5 as a COMMD1-associated} factor

Using tandem affinity purification (TAP), we previously identified a number of putative COMMD1-associated factors (Burstein et al. 2005). The HAT GCN5 was tentatively identified among them through a $\mathrm{C}$-terminal peptide detected by LC/MS-MS (Fig. 1A). This interaction was first confirmed through independent coimmunoprecipitation experiments. Precipitation of GST-tagged COMMD1 from cell lysates resulted in the coprecipitation of GCN5, and, furthermore, binding to GCN5 was mapped to the COMM domain, the homology domain that is present in all COMMD proteins (Fig. 1B). Indeed, another COMM domain-containing protein, COMMD10, was also able to interact with GCN5 (Fig. 1C). Furthermore, immunoprecipitation of endogenous COMMD1 resulted in the coprecipitation of endogenous GCN5, and the converse precipitation also demonstrated the presence of an endogenous COMMD1/GCN5 complex in cells (Fig. 1D).

\section{GCN5 inhibits NF-кB-mediated transcription}

Given the role of COMMD1 as an NF-кB inhibitor, we examined whether GCN5 plays a role in the regulation of this pathway. Transfection of 293 cells with siRNA against GCN5 led to exaggerated induction of TNF mRNA in response to TNF stimulation (Fig. 1E); CX3CL1 (fractalkine) and TNFAIP3 (A20) were similarly up-regulated (Supplemental Fig. S1A,B). Conversely, expression of GCN5 by plasmid transfection inhibited the induction of the TNF gene (Fig. 1F). Moreover, concurrent expression of an siRNA-resistant mutant of GCN5 abrogated the effect of the siRNA oligonucleotide on the expression of TNFAIP3 (Supplemental Fig. S1E). Altogether, these data supported the conclusion that these are specific effects of the siRNA oligonucleotide targeting GCN5. Similar experiments were performed in U2OS cells after lentiviral delivery of a shRNA targeting GCN5 and, once again, GCN5deficient cells demonstrated exaggerated expression of NF-кB-regulated genes after TNF stimulation, such as
ICAM1 (Fig. 1G) as well as IL8 and TNF (Supplemental Fig. S1C,D). These data indicated that endogenous GCN5 inhibits several TNF-inducible genes in more than one cell line. To address whether the repressive effect of GCN5 is mediated through NF-кB itself, TNF gene expression was examined after depletion of RelA, a key NF-кB subunit. The efficiency of the RNAi was demonstrated by Western blotting (Supplemental Fig. S2). As predicted, stimulated TNF expression was dampened by RelA deficiency (Fig. 1H, open bars). Interestingly, the exaggerated expression of TNF in GCN5-deficient cells was lost upon concurrent deficiency in RelA (Fig. 1H, black bars), supporting the notion that GCN5 acts on RelA to suppress the TNF gene.

\section{GCN5 interacts with RelA and promotes its ubiquitination}

Given the known interaction between COMMD1 and RelA, we investigated whether GCN5 similarly interacts with RelA. Immunoprecipitation of endogenous GCN5 resulted in the recovery of RelA from whole-cell lysate (WCL) or nuclear extracts, and, in both instances, TNF stimulation resulted in greater coprecipitation (Fig. 2A). Next, we resorted to a sequential affinity purification scheme to examine whether purified GCN5/COMMD1 complexes could interact with endogenous RelA. As expected, GCN5 bound both COMMD1 and RelA (Fig. 2B, PD: GCN5), and importantly, the purified GCN5/ COMMD1 complex also contained endogenous RelA (Fig. 2B, Re-PD: COMMD1).

The interaction between GCN5 and COMMD1 and their shared ability to bind to and inhibit NF- $\kappa$ B suggested that these two proteins might work through a common mechanism. Hence, we examined if, similar to COMMD1, GCN5 could promote RelA ubiquitination in the nucleus. Nuclear extracts were prepared and endogenous RelA was precipitated after denaturing the lysate by adding SDS and boiling, and the recovered material was immunoblotted for polyubiquitin. Expression of GCN5 in 293 cells resulted in greater amounts of ubiquitinated RelA (Fig. 2C, right), while the level of ubiquitinated RelA was decreased in U2OS cells deficient in GCN5 (Fig. 2C, left). This finding was confirmed by another approach, where cells were transfected with $\mathrm{His}_{6}$-tagged Ubiquitin and ubiquitinated RelA was detected after precipitating ubiquitinated proteins with nickel agarose beads. As shown in Supplemental Figure S3A, decreased GCN5 expression also led to decreased recovery of ubiquitinated RelA by this approach. Moreover, GCN5 deficiency impaired the ability of COMMD1 to promote RelA ubiquitination (Supplemental Fig. S3B), suggesting that GCN5 is a required cofactor for COMMD1-mediated ubiquitination, an effect that is congruent with its interaction with COMMD1.

\section{GCN5 controls nuclear RelA stability and its interaction with promoter sites}

Previous studies indicate that RelA ubiquitination is necessary for the proper termination of RelA-promoter 
A.

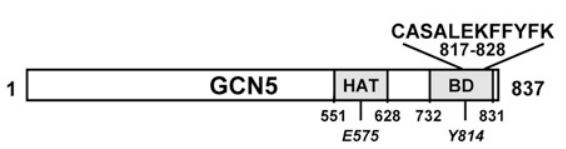

B.

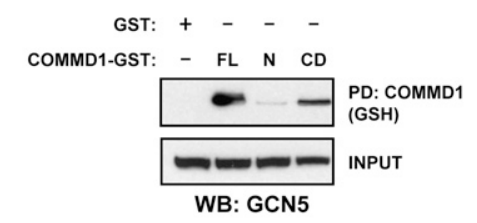

C.

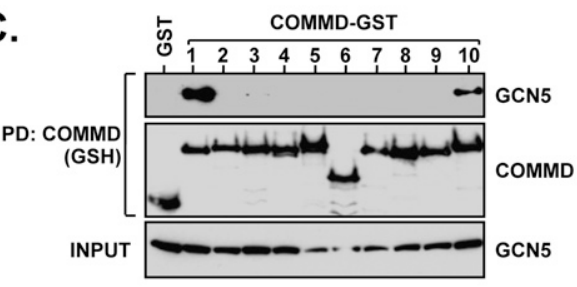

E.

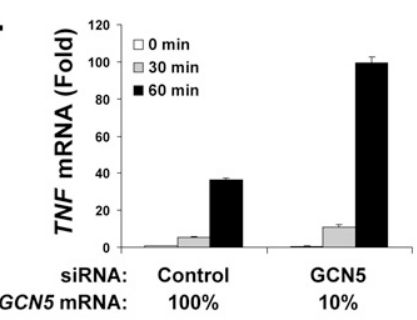

G.

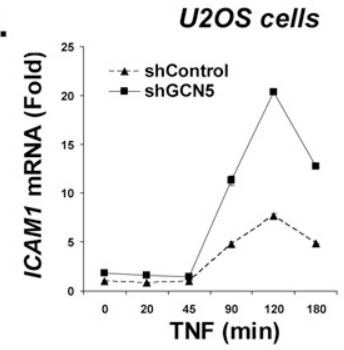

D.
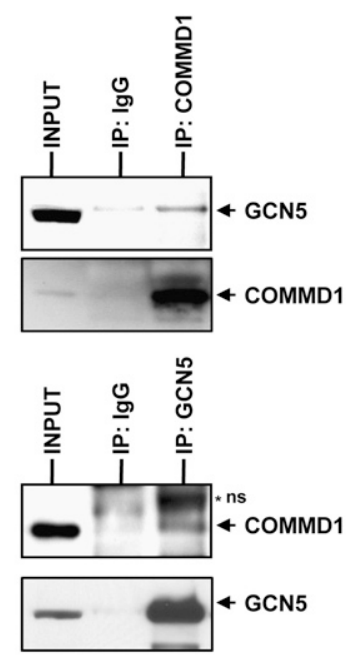

F.

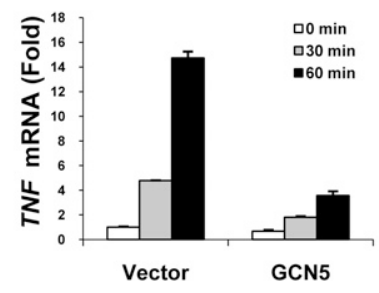

Figure 1. GCN5 binds to COMMD1 and inhibits NF-кB-mediated transcription. $(A)$ Identification of GCN5 as a COMMD1associated factor. Schematic representation of GCN5 with the boundaries of its HAT domain and Bromo domain (BD). The C-terminal peptide of GCN5 (amino acids 817-828) identified by LC/MS-MS is shown. The HAT activity and Bromo domain function are dependent on residues E575 and Y814, respectively. $(B)$ COMMD1 binds GCN5 through its COMM domain. GCN5 was coexpressed with full-length COMMD1 $(\mathrm{FL})$, its $\mathrm{N}$ terminus $(\mathrm{N})$, or its COMM domain (CD) fused with GST. Subsequently, COMMD1 was precipitated and the recovered material was immunoblotted for GCN5. (C) GCN5 interacts with other COMMD proteins. HEK293 cells were cotransfected with GCN5 and the indicated COMMD proteins fused to GST, which were subsequently precipitated from cell lysates using GSH Sepharose beads. The presence of coprecipitated GCN5 was determined by immunoblotting. (D) Coprecipitation of endogenous GCN5 and COMMD1. Cell extracts were subjected to immnoprecipitation for COMMD1 (top panel) or GCN5 (bottom panel). Immunoblotting for GCN5 and COMMD1 was performed as indicated (ns, nonspecific band; input is $0.5 \%$ of the IP material). (E-G) GCN5 represses NF-кB-dependent gene expression. HEK293 cells were transiently transfected with siRNA against GCN5 $(E)$ or a GCN5 expression vector. U2OS cells were stably transduced with a lentivirus expressing shRNA against GCN5 $(F)$. After TNF stimulation, the indicated transcript levels were measured by qRT-PCR. (H) The effect of GCN5 is dependent on RelA. HEK293 cells were transiently transfected with siRNA against GCN5, and additionally, cells received control siRNA transfection (open bars) or siRNA transfection against RelA (black bars). The effect on TNF transcript levels was assessed by qRT-PCR.

interactions (Saccani et al. 2004). Consistent with this, COMMD1 deficiency is associated with prolonged RelA binding to promoter sites (Burstein et al. 2005) and leads to blunted disappearance of RelA from the nucleus (Maine et al. 2007). Therefore, we examined whether GCN5 deficiency would similarly result in both of these events. First, nuclear levels of RelA in GCN5-deficient U2OS cells were compared with the corresponding control. IкB resynthesis and nuclear export, a negative feedback loop in the NF-кB pathway, was inhibited by cycloheximide (CHX). As shown in Figure 2D, GCN5 deficiency led to greater RelA nuclear levels, particularly at late time points after TNF stimulation. Next, we examined RelA recruitment to the ICAM1 and IL8 promoters by chromatin immunoprecipitation (ChIP) and found that in both instances, GCN5 deficiency led to prolonged promoter occupancy by RelA (Fig. 2E). In addition, these experiments demonstrated that GCN5 was inducibly recruited to these promoter sites. Altogether, the data indicated that akin to COMMD1, GCN5 participates in controlling the levels of nuclear and chromatin-associated RelA.

The HAT activity of GCN5 is dispensable for its role in RelA degradation

RelA can be acetylated by p300 (Chen et al. 2001, 2002), and the GCN5 homolog PCAF has also been reported to acetylate RelA (Kiernan et al. 2003). Therefore, we evaluated whether GCN5-promoted RelA ubiquitination could be linked to RelA acetylation. While p300 promoted significant accumulation of acetylated RelA, neither GCN5 nor PCAF had an appreciable effect (Supplemental Fig. S4). Nevertheless, the HAT activity of GCN5 could 
Mao et al.

Figure 2. GCN5 interacts with RelA and promotes its ubiquitination. (A) Coprecipitation of endogenous RelA and GCN5. WCLs or nuclear extracts were prepared from unstimulated and TNF-stimulated HEK293 cells. GCN5 was immunoprecipitated, and the recovered material was immunoblotted for RelA (input is $0.8 \%$ of the IP material). (B) The GCN5/ COMMD1 complex interacts with endogenous RelA. GCN5 fused with GST and COMMD1 fused to a biotinylation tag were expressed in cells. GCN5 was purified through a GSH affinity column (PD: GCN5); GCN5 was eluted from the column and COMMD1 was precipitated from this fraction using streptavidin-agarose beads (Re-PD: COMMD1). Western blots for endogenous RelA, GCN5, and COMMD1 are presented. $(C)$ GCN5 promotes the ubiquitination of endogenous RelA. Nuclear extracts from U2OS GCN5-deficient cells (left panels) or HEK293 cells transfected with GCN5 (right panels) were prepared. A denatured immunoprecipitation for RelA was subsequently performed and the presence of ubiquitinated RelA was determined by immunoblotting for ubiquitin. The deficiency and overexpression of GCN5 were determined by Western blotting for GCN5. (D) GCN5 deficiency increases nuclear RelA level. The GCN5deficient U2OS cell line or the corresponding control line were treated with TNF for $10 \mathrm{~min}$, followed by cycloheximide (CHX). At the indicated time points, cells were harvested and nuclear extracts were prepared and immunobloted for RelA and RNA Pol II (as a loading control). (Bottom panel) I $\kappa$ B- $\alpha$ levels in cytosolic extracts were determined by Western blotting to monitor the effect of TNF and CHX. (E) GCN5 deficiency prolongs RelA binding to NF-кB-responsive promoters. The same GCN5-deficient and control U2OS cell lines were treated with TNF as before. At the indicated time points, cells were lysed and used for ChIP. Occupancy of RelA, GCN5, and RNA Pol II on the ICAM1 and IL8 gene promoters was analyzed. $(F)$ GCN5 promotes RelA ubiquitination despite inactivating mutations in its HAT or Bromo domains. GCN5 wild-type, E575Q (HAT-deficient), or Y814A (Bromo domain-deficient) were cotransfected with HA-RelA and His ${ }_{6}$-tagged ubiquitin, and the levels of ubiquitinated RelA were determined as before. $(G)$ GCN5 promotes the degradation of RelA independently of its HAT activity. HEK293 cells were transfected with RelA along with GCN5 wild-type or E575Q mutant, in combination with COMMD1 or Cul2 as indicated. Cells were lysed and the expression of RelA was determined by Western blot. $(H)$ The HAT activity of GCN5 is dispensable for repression of $\kappa \mathrm{B}$-dependent transcription. The effects of GCN5 E575Q on an NF-kB-responsive reporter (3кB-luc) or a Smad-responsive reporter (SBE-JONK) were examined by luciferase assay.

be more broadly involved in RelA ubiquitination, perhaps acting on a different substrate. To test this notion, we introduced an E575Q mutation in GCN5 to inactivate its HAT domain (Tanner et al. 1999) or a Y814A point mutation that disrupts acetylated-lysine recognition by its Bromo domain (Hudson et al. 2000). Interestingly, neither point mutation significantly impaired GCN5mediated RelA ubiquitination (Fig. 2F). Indeed, the HAT-deficient point mutant E575Q promoted the degradation of RelA to a similar extent as wild-type GCN5 (Fig. $2 \mathrm{G}$ ) and it inhibited RelA-mediated activation of an NFкB-responsive reporter plasmid, whereas it did not affect the activity of a Smad-responsive promoter (Fig. 2H).
Altogether these results indicated that GCN5-promoted RelA ubiquitination is separable from its known HAT activity or the function of its Bromo domain, establishing this effect as a separable novel property of GCN5.

\section{GCN5 interacts with the COMMD1-containing ligase}

COMMD1 promotes RelA ubiquitination through its interaction with a Cul2-containing ubiquitin ligase (Maine et al. 2007), suggesting that GCN5 could also interact with this complex. To investigate this possibility, GCN5 expressed in mammalian cells was precipitated from cell lysates and offered to an in vitro reaction 
containing the E1 and E2 (UbcH5a) enzymes, recombinant ubiquitin, and an ATP regenerating buffer. Ubiquitin ligase activity, evident by polyubiquitin chain formation in vitro, was readily recovered. In contrast, recombinant GCN5 prepared in Escherichia coli was devoid of activity (Fig. 3A). However, when recombinant GCN5 was mixed with cellular lysates and subsequently extensively washed, ubiquitin ligase activity was reconstituted, suggesting that that the activity detected was secondary to the association of GCN5 with other cellular factors. Interestingly, its interaction with the substrate, RelA, was not responsible for precipitating this activity, as this was evident when using lysates from rela-deficient fibroblasts (Supplemental Fig. S5).

We were particularly interested in knowing whether GCN5 could interact with Cul2 complexes, given the role of Cul2 as the main scaffold subunit of the COMMD1containing ligase that targets RelA. To address this question, Cul2 complexes were purified from cells, and expected partner proteins such as COMMD1 and Elongin $\mathrm{C}$ were identified in this material (Fig. 3B). Importantly, GCN5 was also copurified, while TAF6, a component of GCN5-containing coactivator complexes such as TFTC and STAGA, was absent. Additionally, the converse precipitation of GST-GCN5 from cell lysates also coprecipitated endogenous Cul2 (Fig. 3C) and this was not affected by TNF stimulation (Supplemental Fig. S6). Interestingly, the recovered Cul2 was predominantly the active neddylated form, which is enriched in the cell nucleus, where GCN5 resides (Maine et al. 2007).

Collectively, these data supported the notion that GCN5 does not have intrinsic ligase activity, but interacts with an active Cul2/COMMD1 ubiquitin ligase. However, it was recently reported that PCAF, a close homolog of GCN5, possesses intrinsic ubiquitin ligase activity in its $\mathrm{N}$ terminus, a region that is conserved between the two proteins (Linares et al. 2007). Therefore, we examined which region in GCN5 is responsible for its interaction with ubiquitin ligase components. The $\mathrm{C}$ terminus of GCN5 (HAT/Bromo, amino acids 491-837) was found to mediate its interactions with COMMD1 and Cul2 (Fig. 3D), and, in contrast to PCAF, this domain, and not the $\mathrm{N}$ terminus, was able to provide ligase activity in vitro (Fig. 3E).

\section{RelA phosphorylation enhances its binding to GCN5}

While we had determined that GCN5 promotes RelA ubiquitination, a unique role for GCN5 in this process was not yet apparent. To address this question, we examined in more detail the GCN5-RelA interaction, and the mechanism behind its TNF-inducible nature (Fig. $2 \mathrm{~A}, \mathrm{~B})$. First, cells transfected with GCN5 were treated with various IKK-activating stimuli, namely TNF, IL1- $\beta$, or Flagellin, and the formation of the GCN5-RelA complex was assessed by coprecipitation of endogenous RelA. All of these forms of cell stimulation resulted in increased recovery of RelA (Fig. 4A). This suggested that the inducible formation of the GCN5-RelA complex could be a downstream consequence of IKK activation, such as the translocation of RelA into the nucleus, where GCN5 resides. Therefore, we first examined whether the effect of TNF could be recapitulated under in vitro conditions, where the cellular redistribution of RelA would not be a factor. Cell lysates were prepared after TNF stimulation and offered to bacterially made recombinant GCN5-GST for in vitro binding. After precipitation of GCN5, endogenous RelA could be easily recovered, and, importantly, TNF stimulation prior to lysis enhanced the ability of GCN5 to bind to RelA (Fig. 4B). Since the interaction in this experiment occurred post-lysis, the effect of TNF could not be explained by cellular redistribution of RelA.

Another consequence of IKK activation is IкB degradation, and, therefore, we speculated that perhaps ІкB might regulate GCN5-RelA complex formation. To examine this notion, cells were transfected with IкB- $\alpha$ superdominant (SD), a stable mutant that lacks the IKK phosphorylation sites. As shown in Figure 4C, the interaction between GCN5 and RelA was not impaired by IкB- $\alpha$ SD expression and, in fact, this protein was incorporated into the GCN5-RelA complex, indicating that ІкB proteins do not impair the GCN5-RelA interaction.

Finally, we examined whether phosphorylation of RelA itself might be responsible for its increased binding to GCN5. To this end we repeated the in vitro binding experiment after treating cells with Calyculin A, a phosphatase inhibitor that causes dramatic accumulation of phosphorylated RelA (Supplemental Fig. S7). Exposure to this agent prior to cell lysis resulted in very dramatic enhancement of RelA-GCN5 binding in vitro (Fig. 4D). Importantly, treatment of cellular lysates with recombinant $\lambda$-protein phoshatase ( $\lambda$-PPase) prior to incubation with GCN5 reversed the binding of RelA to GCN5 to the unstimulated level (Fig. 4E).

\section{RelA phosphorylation is linked to its ubiquitination and degradation}

Since RelA phosphorylation enhances its binding to GCN5, phosphorylation would be expected to trigger RelA ubiquitination. Indeed, prior reports indicate that LPS and other TLR ligands that promote IKK activation and RelA phosphorylation also accelerate its ubiquitination (Tanaka et al. 2007). We examined this notion by determining the effect of Calyculin A on the ubiquitination of RelA in vivo, and found that this agent promoted significant accumulation of ubiquitinated RelA (Fig. 4F). In addition, phosphorylated RelA accumulated more dramatically when a proteasomal inhibitor was also used (Fig. 4G), consistent with the notion that phosphorylated RelA is unstable and undergoes ubiquitination and proteasomal degradation. Finally, we examined the effect of Calyculin A on the ubiquitination of RelA in vitro. To this end, GCN5 was expressed and subsequently precipitated from cell lysates and offered as a source for ligase activity in vitro. Coprecipitation of endogenous RelA was observed when this material was immunoblotted for RelA. Interestingly, high molecular weight material accumulated after the ubiquitination reaction in the 
Mao et al.
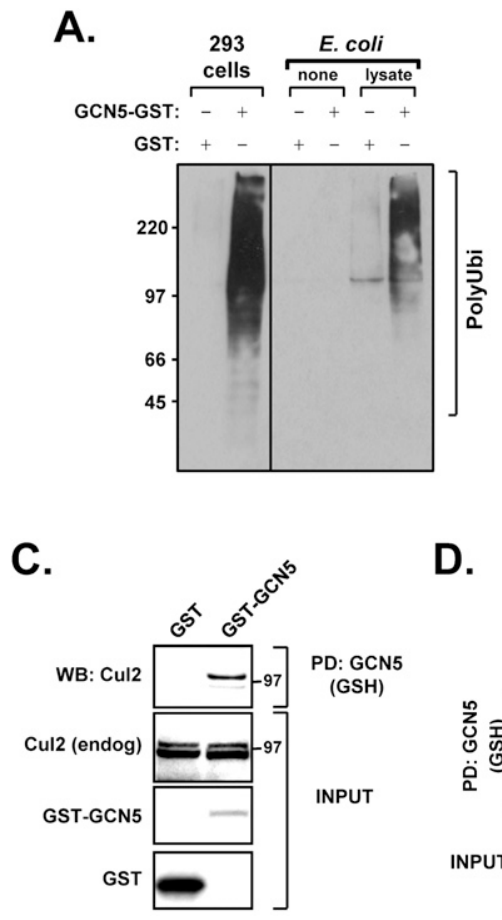

B.

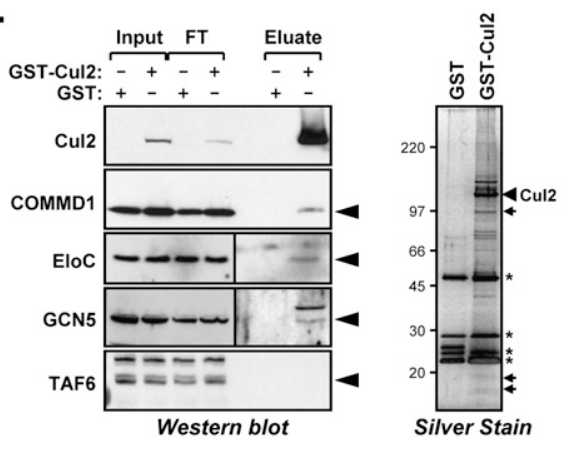

E.

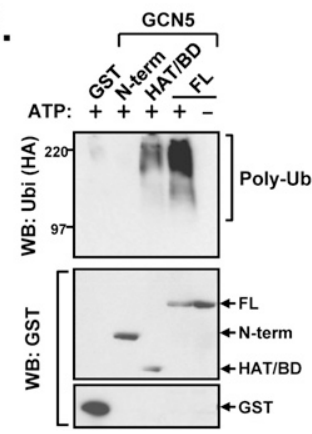

Figure 3. GCN5 interacts with the COMMD1-containing ligase. (A) GCN5 precipitates ubiquitin ligase activity. GCN5-GST or GST precipitated from transfected HEK293 cells, or recombinant (r) GST, or GCN5-GST prepared in E. coli were added to an in vitro ubiquitination reaction. In addition, rGST or rGCN5-GST were mixed with an HEK293 cell lysate, incubated at $4^{\circ} \mathrm{C}$ for $2 \mathrm{~h}$, and then extensively washed prior to being used as a source for ubiquitin ligase activity. Formation of polyubiquitin chains in the reaction was determined by SDS-PAGE and immunoblotting for ubiquitin. (B) GCN5 was copurified as a Cul2-associated factor. Lysates were prepared from HEK293 cells overexpressing a GST-Cul2 fusion protein (Input) and were applied to a GSH-Sepharose affinity column, from which Cul2-containing complexes were subsequently eluted and concentrated by filtration (Eluate). The input, the flow-through material not bound to the column (FT), and eluate were subjected to immunoblotting for Cul2, COMMD1, Elongin C (EloC), GCN5, and TAF6. Silver stain of the eluate is shown in the right panel with the bands corresponding to Cul2, and the immunoblotted subunits noted by small arrows. Nonspecific bands are indicated by an asterisk $\left({ }^{\star}\right)$. $(C)$ GCN5 binds to Cul2, the main scaffold protein of the COMMD1-containing ligase. HEK293 cells were transfected with GST or GST-GCN5, which were precipitated from cell lysates. The presence of coprecipitated endogenous Cul2 was determined by Western blot. The position of the 97-kDa molecular weight marker is indicated. $(D)$ The C terminus of GCN5 binds to COMMD1 and Cul2. Cells were transfected with GCN5 full-length (FL) fused to GST or the indicated truncation mutants along with Flag-Cul2 or COMMD1-Flag. GCN5 was precipitated by GSH Sepharose beads, and the presence of Cul2 or COMMD1 in the precipitated material was determined by Western blot analysis. $(E)$ The C terminus of GCN5 containing the HAT and Bromo domains precipitates E3 ligase activity. GCN5 full-length (FL) fused to GST or truncation mutants spanning its $\mathrm{N}$ terminus ( $\mathrm{N}$-term, amino acids 1-491) or $\mathrm{C}$ terminus (HAT/Bromo, amino acids 492-837) were expressed in mammalian cells. These proteins were precipitated from cell lysates, added to in vitro ubiquitination reactions, and polyubiquitin chain formation was determined as before.

presence of ATP (Fig. 4H). This was particularly evident when the cells had been treated with Calyculin A prior to lysis, with the caveat that this treatment also increased the amount of RelA that coprecipitates with GCN5. Altogether, the data indicated that GCN5-associated ligase activity can ubiquitinate RelA, particularly when it is phosphorylated. This is consistent with the observed propensity of phosphorylated RelA to bind to GCN5 and to be ubiquitinated and degraded in cells.

\section{RelA S468 phosphorylation promotes GCN5 binding}

We next turned our attention to the mechanism responsible for RelA-GCN5 complex formation by examining the domain in RelA responsible for GCN5 binding. In vitro translated radiolabeled GCN5 bound avidly to the transactivation domain of RelA (TAD, spanning amino acids 305-551) (Fig. 5A), in contrast to COMMD1, which binds to the N-terminal end of the RHD (Burstein et al. 2005; Maine et al. 2007). Similar results were observed when precipitating RelA truncation mutants expressed in human embryonic kidney (HEK) 293 cells (Supplemental Fig. S8A). In addition, binding between GCN5 and the TAD of RelA was similarly inducible by RelA phosphorylation and reversible by dephosphorylation with $\lambda$-PPase (Fig. 5B), indicating that the phosphorylation site(s) responsible for inducible binding might be contained within the TAD of RelA.

Further in vitro binding experiments indicated that the last 21 amino acids that constitute the first transactivation domain (TA1) within the TAD (Schmitz and Baeuerle 1991) are critical for GCN5 binding (Fig. 5C, RelA 1-530), 
A.

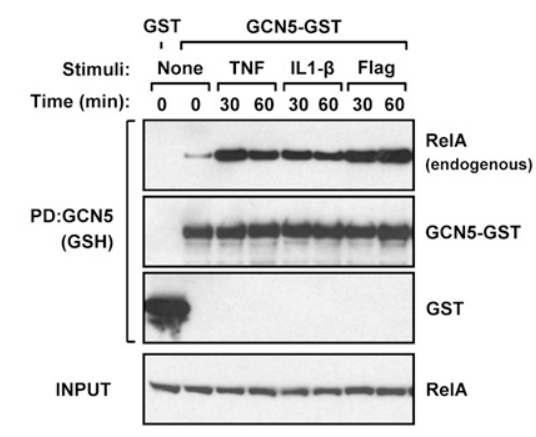

C.

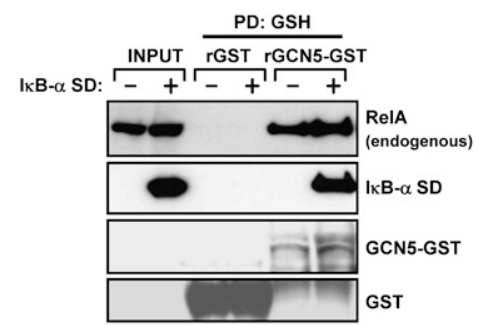

E.

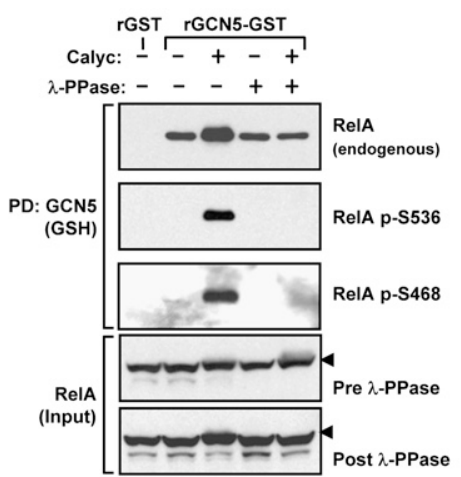

B.

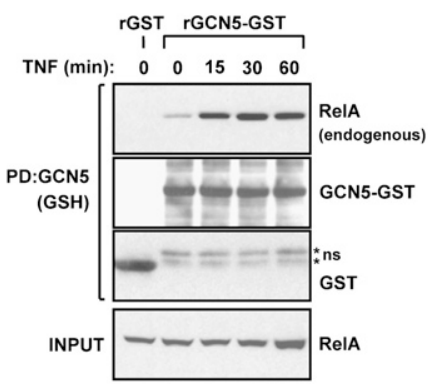

D.

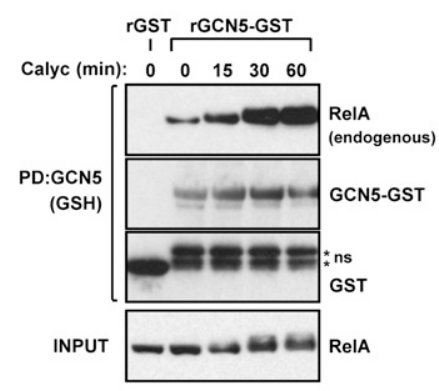

F.
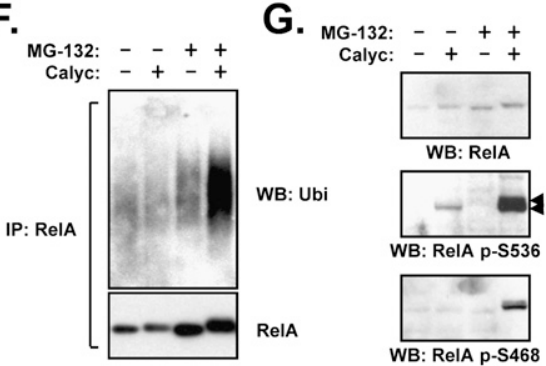

H.

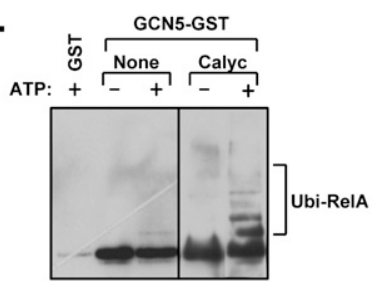

Figure 4. RelA phosphorylation enhances GCN5-RelA interactions. (A) Various IKKactivating stimuli promote GCN5-RelA binding. HEK293 cells were transfected with GCN5-GST or GST and subsequently treated with TNF, IL-1 $\beta$, or Flagellin prior to lysis and GSH precipitation. The presence of coprecipitated endogenous RelA was determined by immunoblotting. $(B)$ TNF treatment prior to lysis promotes RelA-GCN5 binding in vitro. HEK293 cells were treated with TNF or Calyculin A, and the obtained lysates were mixed with bacterially made rGST or rGCN5-GST, followed by precipitation. The presence of endogenous RelA in the recovered material was determined by immunoblotting. $(C)$ IкB does not impair the GCN5-RelA interaction. HEK293 cells were transfected with IкB- $\alpha$ superdominant $(\mathrm{SD})$, and the ability of recombinant GCN5 to precipitate endogenous RelA post-lysis was examined as in $B .(D)$ The phosphatase inhibitor Calyculin A promotes RelA-GCN5 binding in vitro. HEK293 cells were treated with Calyculin A and the obtained lysates were mixed with rGST or rGCN5-GST, followed by precipitation. The presence of endogenous RelA in the recovered material was determined by immunoblotting. $(E)$ Dephosphorylation of RelA abrogates its induced binding to GCN5. HEK293 cells were treated with Calyculin A and subsequently lysed in a buffer without phosphatase inhibitors. The lysate was incubated with $\lambda$-protein phosphatase $(\lambda$ PPase) as indicated, and in vitro binding of RelA to recombinant GCN5 was performed as in $D$. The recovered material was immunoblotted to detect the coprecipitation of endogenous RelA and phosphorylated RelA. $(F)$ Phosphorylation promotes RelA ubiquitination. Wild-type MEFs were treated with MG-132 (30 min) and Calyculin A as indicated. RelA was subsequently immunoprecipitated from cell lysates and the presence of ubiquitinated RelA was determined by immunoblotting for ubiquitin. (G) Phosphorylated RelA is labile and is stabilized by proteasomal blockade. Wild-type MEFs were treated with Calyculin A and MG-132 as indicated. Phosphorylated RelA levels were determined by Western blot using two phospho-specific antibodies. $(H)$ GCN5-associated ligase activity ubiquitinates RelA in vitro. HEK293 cells transfected with GCN5-GST or GST were treated without or with Calyculin A as shown. Material precipitated by GSH beads was applied to an in vitro ubquitination reaction as in Figure 3A. Coprecipitated RelA and its ubiquitination were shown by immunoblotting.

and similar results were obtained in coprecipitation experiments using mammalian cell lysates (Supplemental Fig. S8B). Additionally, a recombinant peptide prepared in E. coli and spanning most of the TA1 region (amino acids 531-547) was capable of completely disrupting the RelA-GCN5 complex (Fig. 5D), consistent with the notion that TA1 is a major interface for RelA-GCN5 binding. Interestingly, TA1 contains Ser 536, a major phosphorylation site in RelA that has been implicated previously in the stability of this protein (Lawrence et al. 2005). Therefore, we initially speculated that perhaps Ser
536 might be responsible for phosphorylation-dependent binding between RelA and GCN5. Indeed, loss of the last 21 amino acids of RelA abrogated all inducible binding between RelA-TAD and GCN5 (Fig. 5E, $\Delta 21$ ). However, point mutations of any of the six serines located in this region, including Ser 536, did not have a substantial effect on binding (data not shown). Surprisingly, even a combined mutation of all six serines in TA1 did not abrogate the inducible binding between RelA-TAD and GCN5 (Fig. 5E, 6S/A), suggesting that the phosphorylation site might be located elsewhere in the TAD. Indeed, examination 
Mao et al.

Figure 5. The GCN5-RelA interaction requires the $\mathrm{C}$ terminus of RelA and is enhanced by phosphorylation at Ser 468 . (A) GCN5 interacts with the transactivation domain of RelA. Recombinant RelA fragments fused to GST were purified from E. coli and their ability to precipitate radiolabeled in vitro translated GCN5 was examined by autoradiography. Coomasie staining demonstrates the amount of recombinant protein used. $(B)$ The TAD of RelA is responsible for phosphorylation-inducible binding. The same experiment as in $B$ was performed, but this time using lysates from cells transfected with RelA-TAD (306-551). (Bottom panel) In addition, $\lambda$ PPase treatment of the lysate was also performed prior to binding. $(C)$ GCN5 interacts with TA1, encompassed by the last 21 amino acids of RelA. The indicated recombinant RelA fragments fused to GST were purified from HEK293 cells, and their ability to precipitate radiolabeled in vitro translated GCN5 was examined by autoradiography. Coomasie staining demonstrates the amount of recombinant protein used. (D) A peptide from TAl is capable of disrupting GCN5-RelA binding in vitro. Recombinant RelA fragments fused to GST were purified from E. coli, and their ability to precipitate radiolabeled in vitro translated GCN5 was examined as in A. In addition, peptides spanning the indicated amino acid residues in RelA were added to the in vitro binding reaction as potential competitive inhibitors. (E) The phosphorylation residue that mediates RelA-GCN5 inducible binding is not contained in the last 21 amino acids of RelA. The same experiment as in Figure 4E, but this time using two mutations in the TAD: deletion of the last 21 amino acids (306-530), $\Delta 21$; or mutation of the last six serines in RelA (S535, 536, 543, 547, 550, 551A), 6S/A. (F) Ser 468 phosphorylation is required for RelAGCN5-inducible binding. The same experiment as in $B$ and $E$, but using either Calyculin A treatment (left) of TNF stimulation (right). Wild-type RelA or a point mutant at Ser 468 are compared.

of other serine phosphorylation sites within RelA indicated that mutation of Ser 468 abrogated the inducible binding between RelA and GCN5 in response to either Calyculin A or TNF treatments (Fig. 5F). Collectively, these data suggested that the last 21 amino acids of RelA provide the main domain for GCN5 binding, and that upon phosphorylation of RelA at Ser 468, additional conformational changes take place that increase its binding to GCN5.

\section{IKK is required for RelA-GCN5 complex formation}

Previous reports indicate that IKK $\beta$ or IKK $\varepsilon$ can phosphorylate RelA on Ser 468 (Schwabe and Sakurai 2005; Mattioli et al. 2006), while other studies have linked IKK $\alpha$-mediated phosphorylation of RelA to its proteasomal degradation (Lawrence et al. 2005). Hence, we exam- ined the possible participation of the IKK complex as the kinase for RelA that promotes GCN5 binding. To address this question, we used Ikk-deficient mouse embryo fibroblasts (MEFs) to examine the binding between RelA and GNC5 in vitro (Fig. 6A). TNF treatment prior to lysis induced RelA-GCN5 binding when lysates were prepared from wild-type fibroblasts, while loss of $I k k \alpha$ or $I k k \beta$ completely abrogated the effect of TNF. These data indicated that an intact Ikk complex is required for inducing the binding between RelA and GCN5, likely because of the role of this kinase complex in Ser 468 phosphorylation.

\section{IKK and GCN5 are required for inducible RelA degradation}

If the IKK complex promotes RelA-GCN5 binding, one predicted outcome would be that IKK activation should 
A.

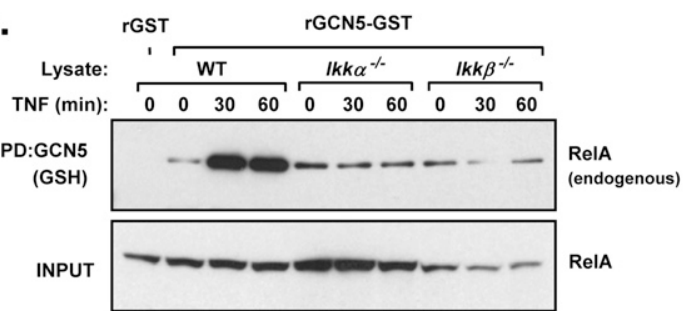

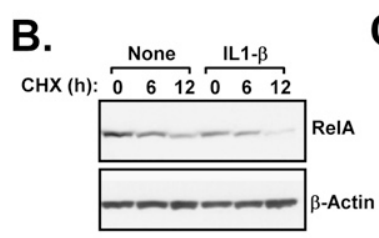

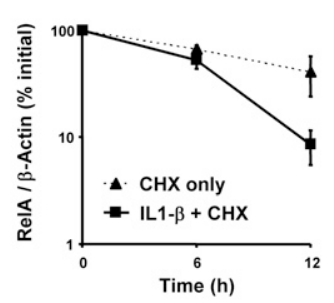

C.

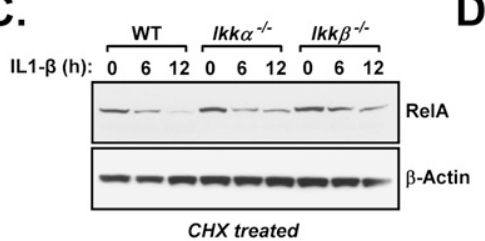

CHX treated

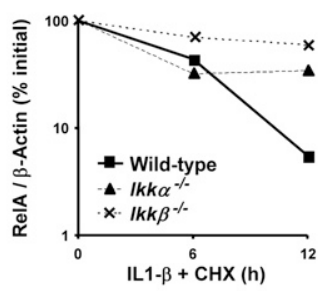

D.

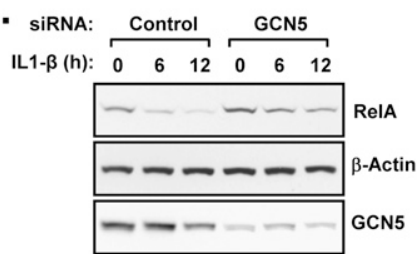

CHX treated

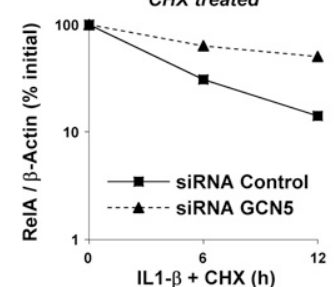

E.
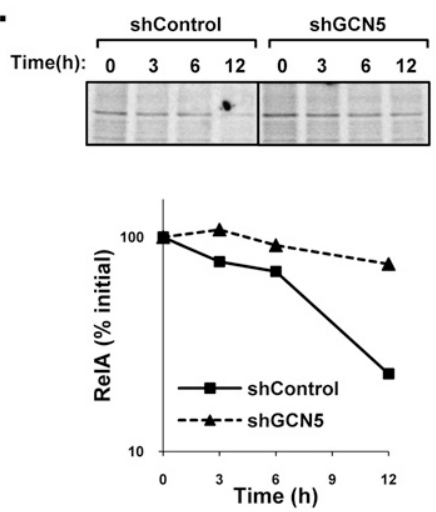

F.

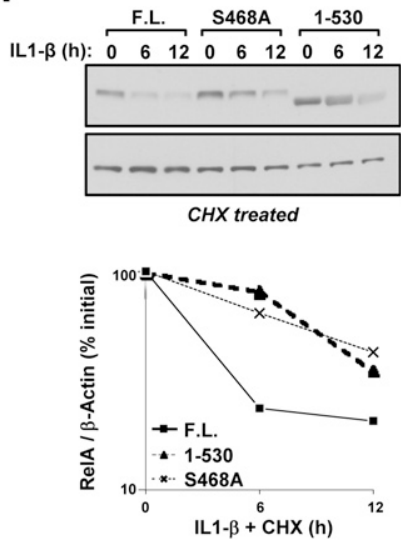

Figure 6. IKK and GCN5 promote RelA degradation. (A) IKK is required for TNF-induced RelA-GCN5 binding. Wild-type (WT), $I k k \alpha^{-/-}$, or Ikk $\beta^{-/-}$MEFs were stimulated with TNF and subsequently lysed. This material was applied to rGCN5-GST for in vitro binding as before. The coprecipitation of endogenous RelA was determined by immunoblotting. (B) IL1- $\beta$ stimulation decreases RelA stability. Cycloheximide (CHX) was used to determine the stability of RelA in wild-type MEFs that were untreated or stimulated with IL1- $\beta$. RelA levels were determined by immunoblotting and densitometry analysis. (C) Ikk deficiency stabilizes RelA in IL1- $\beta$ treated cells. Similar to $B$, but the effects of IL1- $\beta$ treatment were compared between wild type, Ikk $\alpha^{-1-}$, or Ikk $\beta^{-1-}$ MEFs. $(D)$ GCN5 deficiency prevents IL1- $\beta$-promoted degradation. HEK293 cells were transfected with the indicated siRNA and subsequently treated with IL1- $\beta$. The stability of RelA after cycloheximide (CHX) treatment was examined by immunoblotting and densitometry analysis. (E) GCN5 deficiency stabilizes RelA in U2OS cells. GCN5-deficient U2OS cells and the corresponding control cells were metabolically labeled by ${ }^{35} \mathrm{~S}$-methionine and ${ }^{35} \mathrm{~S}$-cysteine. At the indicated time points after labeling, cells were lysed for a denatured immunoprecipitation for RelA. The recovered material was examined by autoradiography after SDS-PAGE separation. $(F)$ RelA 1-530 and RelA S468A are more stable than full-length (FL) RelA. The indicated human RelA constructs were introduced into rela-deficient MEFs through lentiviral infection and selection. Protein stability was examined as before.

destabilize RelA. Using IL1- $\beta$ as a means of activating the kinase, we found that this led to decreased stability of RelA in wild-type fibroblasts compared with unstimulated cells (Fig. 6B). In addition, and consistent with the role of IKK in RelA phosphorylation, the protein was stabilized in Ikk-deficient fibroblasts treated with IL1- $\beta$ (Fig. 6C). Similar to MEFs, cytokine stimulation resulted in a shorter half-life of RelA in 293 cells (data not shown), and most importantly, GCN5 deficiency greatly stabilized RelA in this context (Fig. 6D), akin to the effect of 
IKK deficiency. The stabilization of RelA was similarly observed in pulse-chase metabolic labeling studies examining GCN5-deficient U2OS cells (Fig. 6E). Finally, the stability of mutations in RelA that affect its binding to GCN5, namely a truncation of its TA1 (RelA 1-530) or loss of Ser 468 (S468A), was examined after stably reintroducing these proteins into rela-deficient MEFs. As shown in Figure 6F, these mutants were stabilized compared with the wild-type protein. Altogether, these findings are consistent with the role of IKK-mediated phosphorylation in promoting the RelA-GCN5 interaction, which in turn leads to RelA ubiquitination.

\section{Discussion}

An important phase in the regulation of $\kappa \mathrm{B}$-dependent transcription is the termination of NF- $\mathrm{kB}$ activity, which has been largely ascribed to NF-кB-mediated resynthesis of IкB proteins (Hoffmann et al. 2002). Recently, ubiquitination of NF- $\mathrm{BB}$ has been found to be an additional mechanism by which transcriptionally active NF- $\mathrm{B}$ is suppressed (Saccani et al. 2004). One of the ligases responsible for this effect contains COMMD1 in association with a multimeric complex that contains Cul2 (Ryo et al. 2003; Maine et al. 2007), while others have identified PDLIM2 in a similar role (Tanaka et al. 2007). Additionally, phosphorylation of RelA by IKK $\alpha$ has been reported to destabilize the protein, and similar to ubiquitination, this controls the chromatin association of RelA (Lawrence et al. 2005). However, since Cul2-containing complexes are not known to engage phospho-serine substrates (Petroski and Deshaies 2005), a link between IKKmediated phosphorylation of RelA and its ubiquitination by this ligase was not evident prior to this study.

This report indicates that GCN5, a HAT best known as an activator of transcription, functions in an unexpected manner in the NF-кB pathway, where it represses transcription by providing a critical link between serine phosphorylation of RelA and its ubiquitination. Various data presented here support a model where GCN5 promotes RelA ubiquitination by interacting directly with both the ubiquitin ligase and RelA in a manner akin to a substrate adaptor protein. Similarly, we conclude that in addition to the well-known core components associated with Cul2 (Ganoth et al. 2001), the ligase uses accessory factors such as COMMD1 and GCN5. Interestingly, the HAT activity of GCN5 is dispensable in this setting, as the point mutant GCN5 E575Q retains the ability to promote RelA ubiquitination and repress NF-кB-dependent transcription. Additionally, the sequential purification of the GCN5/COMMD1 complex suggests that only a small fraction of GCN5 interacts with COMMD1, leading us to speculate that most cellular GCN5 is probably engaged in its well-recognized role in coactivator complexes.

The most interesting property of GCN5 in this pathway is its ability to interact with RelA, preferentially after IKK activation. Our data indicate that phosphorylation of RelA itself is responsible for promoting GCN5RelA binding, as the increased binding between these proteins can be reversed in vitro by treating the lysates with $\lambda$-PPase. This finding is corroborated by mutational analysis, which indicates that phosphorylation of RelA at Ser 468 is required for its inducible binding with GCN5. Interestingly, this phosphorylation event has been reported to have an inhibitory effect on transcription (Buss et al. 2004; Schwabe and Sakurai 2005; Mattioli et al. 2006), and, indeed, RelA S468A promotes greater expression of NF-кB target genes such $I 16$ and Tnf (Supplemental Fig. S9). This finding is consistent with our observation that phosphorylation of Ser 468 facilitates RelA binding to GCN5, a preamble to the ubiquitination and degradation of the protein. In addition, the data also demonstrate that TA1, encompassed in the last 21 amino acids, is critically required for basal GCN5 binding. We speculate that upon Ser 468 phosphorylation, a conformational change in RelA likely takes place that possibly cooperates with TA1 to promote the GCN5RelA interaction. Given that the structure of the TAD of RelA has not been solved, the nature of such conformational change remains unclear. Finally, the data presented here is consistent with prior reports that place these events in the nucleus, particularly on gene promoter sites. Indeed, GCN5, as well as COMMD1, are inducibly recruited to NF-кB-responsive promoters, and their deficiency results in prolonged occupancy of the promoter by RelA, an event that presumably explains the increased transcriptional response.

The participation of GCN5 in the ubiquitination pathway is consistent with genetic evidence that GCN5 has HAT-independent functions. Deficiency of Gcn5 in mice prevents the formation of somites, a neural tube or a notochord, while a HAT-inactivating mutation in the Gcn5 gene results in a milder phenotype (Bu et al. 2007). Finally, our study provides a mechanism to link IKK activation, RelA phosphorylation, and the degradation of DNA-bound, transcriptionally active RelA. In this model, activation of the IKK complex not only initiates NF-кBmediated transcription, but sets in motion a process that ultimately inhibits active NF- $\mathrm{B}$ through the targeting of RelA for GCN5 binding and ubiquitination.

\section{Materials and methods}

Plasmids and siRNA

The plasmids pEBB, pEBG, pEBB-COMMD1-Flag, pEBBCOMMD1-GST FL, N-term (amino acids 1-118), COMM domain (amino acids 119-190), pEBB-Flag-Cul2, pEBG-Cul2 FL pEBG-RelA FL and its truncations 1-305, 1-180, 306-551, pEBB-HA-RelA, pEBB-T7-IкB- $\alpha$ SD, pCW7-His 6 -Myc-Ubiquitin, $3 \kappa \mathrm{K}-\mathrm{luc}, 2 \kappa \mathrm{K}-\mathrm{luc}$, and SBE-JONK have been described previously (Hay et al. 2001; Burstein et al. 2004, 2005; Lewis et al. 2004; Maine et al. 2007). The full-length coding sequence for human GCN5 was obtained from an EST clone (IMAGE clone 5575574) to generate pEBB-GCN5 (untagged). Using this EST clone as template, PCR was used to generate the following plasmids: pcDNA3.1-GCN5， pEBB-Flag-GCN5， pET21b-GCN5-GST, pEBB-GCN5-GST and pEBG-GCN5 FL. Similarly, PCR was used to generate pEBG-GCN5 N-term (amino acids 1-491), pEBGGCN5 HAT/Bromo domain (amino acids 492-837), pEBG RelA 
1-530, pEBB HA- RelA 1-530, pEBG RelA 1-450. pcDNA-Flagp300 was a gift from Dr. Colin Duckett. Expression vectors for point mutants of GCN5 and RelA (pEBB-Flag GCN5 E575Q, Y814A, GCN5 RNAi-resistant, pEBG-RelA S536A, pEBB HARelA TAD S468A, pEBB HA-RelA 6S/A) were created by site directed mutagenesis (Stratagene). The constructs pGEX RelA RHD (1-303) and pGEX RelA TAD (354-551) were used for recombinant protein preparation in $E$. coli as described previously (Ramsey et al. 2008). The plasmid pEBB-TB was generated by amplifying the coding sequence for TEV cleavage site and Biotinylation signal from pFA6a HTB kanMX6 as template, kindly provided by Dr. Kaiser (Tagwerker et al. 2006). The COMMD1 coding sequence was subcloned into this plasmid to generate pEBB-COMMD1-TB. Synthetic siRNA oligonucleotides against Chloramphenicol acetyl transferase (a control target), human GCN5 and human RelA were used, and detailed information about targeting sequences are available upon request. The plasmids pHCMV-VSV-G, pMDLg/pRRE, pRSV Rev, and FG12 (Lois et al. 2002) were used for the generation of recombinant lentiviruses (kindly provided by Dr. David Baltimore). For stable RNAi using lentiviral delivery, we introduced a cassette containing the Histone 1 promoter and a shRNA from pSUPER into the XbaI and XhoI sites of FG12.

\section{Cell culture, transfection, and luciferase assays}

HEK293 cells, HEK 293T cells, and U2OS cells were obtained from American Type Culture Collection and cultured in DMEM supplemented with $10 \%$ FBS and L-glutamine $(2 \mathrm{mM}) . I k k \alpha^{-/-}$, $I k k \beta^{-1-}$, and wild-type MEFs were kindly provided by Dr. Inder Verma (Li et al. 1999a, b). All transient transfection experiments were performed in HEK293 cells using a standard calcium phosphate transfection protocol to transfect plasmids and siRNA (Burstein et al. 2004). In certain experiments, the following agents were applied at the indicated final concentration to the growth media: TNF (Roche, $1000 \mathrm{U} / \mathrm{mL}$ ), IL-1 $\beta$ (Roche, $10 \mathrm{ng} /$ $\mathrm{mL}$ ), Flagellin (Inotek, $50 \mu \mathrm{g} / \mathrm{mL}$ ), Calyculin A (Cell Signaling, 50 $\mathrm{nM}$ ), Cycloheximide (BioVision, $60 \mu \mathrm{g} / \mathrm{mL}$ ), and MG-132 (Boston Biochem, $40 \mu \mathrm{M})$. Luciferase assays were performed as described previously (Burstein et al. 2005).

\section{TAP screening}

The TAP screening performed here has been reported previously (Burstein et al. 2005). Briefly, 293 cells seeded in 15-cm plates were transiently transfected with pEBB-COMMD1-TAP $(15 \mu \mathrm{g}$ of plasmid per plate). Cell lysates were subsequently prepared and applied to a chromatography column containing IgG sepharose beads (GE Healthcare). After a 2-h incubation at $4^{\circ} \mathrm{C}$, the column was drained and washed prior to addition of TEV protease. The eluted material was applied to a chromatography column containing calmodulin 4B beads Healthcare) and incubated for $1 \mathrm{~h}$ at $4^{\circ} \mathrm{C}$. The column was then drained and washed prior to addition of calmodulin elution buffer containing EGTA. The final eluate collected was mixed with cold $10 \%$ Trichloroacetic acid in acetone; after overnight incubation at $-20^{\circ} \mathrm{C}$, precipitated proteins were collected by centrifugation, rinsed in $100 \%$ acetone, and allowed to air dry. These samples were then submitted to the Proteomics Center at the University of Victoria for further processing, including tryptic digestion, HPLC separation, and tandem mass spectrometry (MS/MS) to determine peptide sequences.

\section{Quantitative RT-PCR ( $q R T-P C R)$}

Total RNA was extracted from cells using the RNeasy kit (Qiagen) according to the manufacturer's instructions. An RT reaction with $500 \mathrm{ng}$ of total RNA in $25 \mu \mathrm{L}$ was performed using random hexamers and TaqMan Reverse Transcription Reagents (Applied Biosystems). This was followed by quantitative PCR performed in the 7500 Real-Time PCR system (Applied Biosytems). Oligonucleotides and internal probes for NFKBIA, ICAM1, TNF, and IL8 transcripts were obtained from Applied Biosystems, and a TaqMan PCR Master Mix with GAPDH mRNA quantitation was duplexed in the same well as an internal control. To quantify CX3CL1 mRNA, specific primers spanning over an intron were designed (sequences available upon request). These were combined with SYBERGreen PCR Master Mix for mRNA quantitation and parallel reactions with primers targeting GAPDH were performed as a control for mRNA abundance.

\section{Immunoblotting, immunoprecipitation, and ChIP}

WCLs were prepared by adding Triton lysis buffer $(25 \mathrm{mM}$ HEPES, $100 \mathrm{mM} \mathrm{NaCl}, 10 \mathrm{mM}$ DTT, $1 \mathrm{mM}$ EDTA, 10\% Glycerol, 1\% Triton X-100), RIPA buffer (PBS, 1\% NP-40, 0.5\% Deoxycholate, $0.1 \%$ SDS, $10 \mathrm{mM}$ DTT), or an 8-M urea buffer (8 $\mathrm{M}$ urea, $50 \mathrm{mM}$ Tris at $\mathrm{pH} 8.0,300 \mathrm{mM} \mathrm{NaCl}, 50 \mathrm{mM} \mathrm{NaPO}_{4}$, $0.5 \%$ NP-40) supplemented with $1 \mathrm{mM}$ sodium orthovanadate and protease inhibitors (Roche) as indicated in each experiment. Preparation of cytosolic and nuclear extracts was performed as described previously (Burstein et al. 2005). Immunoprecipitations, GSH precipitations, Ni-NTA precipitations, and immunoblotting were performed as previously described (Burstein et al. 2004, 2005). Densitometry analysis consisted in measurements of integrated density using the Scion Image software. For denatured immunoprecipitation, cells were harvested and lysed initially in Triton X-100 Buffer $(75 \mu \mathrm{L})$. The lysate was then mixed with an equal volume of TSD buffer (50 mM Tris at $\mathrm{pH} 7.5$, $2 \%$ SDS, $5 \mathrm{mM} \mathrm{DTT}$ ) and sonicated for $10 \mathrm{sec}$. Subsequently, the lysate was heated for $5 \mathrm{~min}$ at $80^{\circ} \mathrm{C}$ and Triton X-100 buffer was then added to a final volume of $1500 \mu \mathrm{L}$ (or a final concentration of $0.1 \%$ SDS) prior to conventional immunoprecipitation. For in vitro binding assays, E. coli-expressed GST fusion proteins were loaded onto glutathinone sepharose beads (Burstein et al. 2005) and $2-5 \mu \mathrm{g}$ of recombinant protein was mixed with mammalian cell lysates to coprecipitate its binding partner. The following antibodies were used in our studies: acetylated lysine (Cell Signaling, 9441), $\beta$-Actin (Sigma, A5441), COMMD1 (Burstein et al. 2005), Cul2 (Zymed, 51-1800), Elongin C (BioLegend, 613101), Flag (Sigma, A8592), GST (Santa Cruz Biotechnologies, sc-459), GCN5 (Santa Cruz Biotechnologies, sc-20698, sc-6306), HA (CoVance, MMS101R), IкB- $\alpha$ (Upstate Biotechnologies, 06494), IKK $\alpha$ (Cell Signaling, 2682), IKK $\beta$ (Cell Signaling, 2684), Rbx1 (LabVision, 127-075-160), RelA (Santa Cruz Biotechnologies, sc-372 and sc-8008), RelA phospho-S-536 (Cell Signaling, 3031), RelA phospho-S-468 (Cell Signaling, 3039), ubiquitin (Stressgen, SPA-205), PCAF (Santa Cruz Biotechnologies, sc13124). The TAF6 antibody was a kind gift from Dr. Robert Roeder. ChIP was performed as described previously (Burstein et al. 2005). Details regarding primer sequences are available upon request.

\section{In vitro binding experiments}

GST fusion proteins bound to GSH sepharose were incubated at room temperature with BSA $(1 \mathrm{mg} / \mathrm{mL})$ in $200 \mu \mathrm{L}$ of Z-buffer $(25$ $\mathrm{mM}$ HEPES at $\mathrm{pH} 7.5,12.5 \mathrm{mM} \mathrm{MgCl} 2,150 \mathrm{mM} \mathrm{KCl}, 0.1 \%$ NP$40,20 \%$ glycerol). After $15 \mathrm{~min}$, in vitro translated proteins were added and incubated for another $45 \mathrm{~min}$ at room temperature. Peptides used for competition experiments were prepared as previously as GST-PTD fusion proteins, followed by thrombin 
cleavage as described by the manufacturer (GE Healthcare). The prepared peptides were applied to in vitro binding reactions at a final concentration of $50 \mu \mathrm{M}$. The beads were then washed four times with $1 \mathrm{~mL}$ NETN buffer $(100 \mathrm{mM} \mathrm{NaCl}, 1 \mathrm{mM}$ EDTA, $0.5 \%$ NP-40, $20 \mathrm{mM}$ Tris-Hcl at $\mathrm{pH}$ 8.0). Bound proteins were resolved by SDS-PAGE and subjected to autoradiography. Protein dephosphorylation was performed by incubating cell lysates with $\lambda$-PPase (New England Biolabs) for $30 \mathrm{~min}$ at $30^{\circ} \mathrm{C}$ after addition of the recommended reaction buffer and $\mathrm{Mn}$.

\section{In vitro ubiquitination assays}

Each reaction mixture consisted of recombinant ubiquitin (2.5 $\mu \mathrm{g}$ ), E1 (Ubal, $50 \mathrm{ng}$ ), E2 (UbcH5a, $100 \mathrm{ng}$ ), and ATP regenerating buffer (all obtained from Boston Biochem). These were mixed in reaction buffer $(40 \mathrm{mM}$ HEPES at $\mathrm{pH} 7.9,60 \mathrm{mM}$ potassium acetate, $2 \mathrm{mM}$ DTT, $5 \mathrm{mM} \mathrm{MgCl} 2,10 \%$ glycerol) and incubated for $90 \mathrm{~min}$ at $30^{\circ} \mathrm{C}$ (Maine et al. 2007).

\section{Metabolic labeling}

This was performed as described previously (Maine et al. 2007). Briefly, cells were seeded $24 \mathrm{~h}$ prior to the experiment and then placed for $30 \mathrm{~min}$ in a cysteine and methionine-deficient medium. At the end of this period, ${ }^{35}$ S-radiolabeled methionine and cysteine was added to the medium for $1 \mathrm{~h}$ and then replaced with regular growth medium supplemented with excess nonradiolabeled methionine $(2 \mathrm{mM})$ and cysteine $(2 \mathrm{mM})$. WCLs were then prepared at the indicated time points and the samples were subjected to denatured immunoprecipitation of RelA (Santa Cruz Biotechnologies, sc-372). The recovered material at the end of the immunoprecipitation was resolved by SDS-PAGE and RelA was detected by autoradiography.

\section{Acknowledgments}

We are grateful to Dr. Inder Verma for his generosity in providing the MEFs used in our studies and to Robert Roeder for providing the TAF6 antibody. We also thank Dr. Colin Duckett, Dr. Peter Kaiser, and Dr. David Baltimore for providing various plasmids used here. This work was supported by a VA Merit Review Entry Program Award, a CCFA Senior Research Award, and NIH R01 DK073639 to E.B.; by NCI CA104397 and DOD W81XWH-06-10186 to M.W.M.; and through the University of Michigan's Cancer Center Grant (NIH 5 P30 CA46592).

\section{References}

Arenzana-Seisdedos, F., Turpin, P., Rodriguez, M., Thomas, D., Hay, R.T., Virelizier, J.L., and Dargemont, C. 1997. Nuclear localization of $\mathrm{I} \kappa \mathrm{B} \alpha$ promotes active transport of $\mathrm{NF}-\kappa \mathrm{B}$ from the nucleus to the cytoplasm. J. Cell Sci. 110: 369-378.

Baeuerle, P.A. and Baltimore, D. 1988. I к B: A specific inhibitor of the NF-к B transcription factor. Science 242: 540-546.

$\mathrm{Bu}$, P., Evrard, Y.A., Lozano, G., and Dent, S.Y. 2007. Loss of Gcn5 acetyltransferase activity leads to neural tube closure defects and exencephaly in mouse embryos. Mol. Cell. Biol. 27: 3405-3416.

Burstein, E., Ganesh, L., Dick, R.D., van De Sluis, B., Wilkinson, J.C., Klomp, L.W., Wijmenga, C., Brewer, G.J., Nabel, G.J., and Duckett, C.S. 2004. A novel role for XIAP in copper homeostasis through regulation of MURR1. EMBO J. 23: 244-254.

Burstein, E., Hoberg, J.E., Wilkinson, A.S., Rumble, J.M., Csomos, R.A., Komarck, C.M., Maine, G.N., Wilkinson, J.C., Mayo, M.W., and Duckett, C.S. 2005. COMMD proteins: A novel family of structural and functional homologs of MURR1. J. Biol. Chem. 280: 22222-22232.

Buss, H., Dorrie, A., Schmitz, M.L., Frank, R., Livingstone, M., Resch, K., and Kracht, M. 2004. Phosphorylation of serine 468 by GSK-3 $\beta$ negatively regulates basal p 65 NF-кB activity. J. Biol. Chem. 279: 49571-49574.

Chen, Z., Hagler, J., Palombella, V.J., Melandri, F., Scherer, D., Ballard, D., and Maniatis, T. 1995. Signal-induced site-specific phosphorylation targets IкB $\alpha$ to the ubiquitin-proteasome pathway. Genes \& Dev. 9: 1586-1597.

Chen, L., Fischle, W., Verdin, E., and Greene, W.C. 2001. Duration of nuclear NF- $\mathrm{B}$ action regulated by reversible acetylation. Science 293: 1653-1657.

Chen, L.F., Mu, Y., and Greene, W.C. 2002. Acetylation of RelA at discrete sites regulates distinct nuclear functions of NFкB. EMBO J. 21: 6539-6548.

Ganesh, L., Burstein, E., Guha-Niyogi, A., Louder, M.K., Mascola, J.R., Klomp, L.W., Wijmenga, C., Duckett, C.S., and Nabel, G.J. 2003. The gene product Murrl restricts HIV-1 replication in resting CD4 ${ }^{+}$lymphocytes. Nature 426: 853-857.

Ganoth, D., Bornstein, G., Ko, T.K., Larsen, B., Tyers, M., Pagano, M., and Hershko, A. 2001. The cell-cycle regulatory protein Cks1 is required for $\mathrm{SCF}^{\text {Skp2 }}$-mediated ubiquitinylation of p27. Nat. Cell Biol. 3: 321-324.

Hay, D.C., Kemp, G.D., Dargemont, C., and Hay, R.T. 2001. Interaction between hnRNPAl and I $\mathrm{B} \alpha$ is required for maximal activation of NF-кB-dependent transcription. Mol. Cell. Biol. 21: 3482-3490.

Henkel, T., Machleidt, T., Alkalay, I., Krönke, M., Ben-Neriah, Y., and Baeuerle, P.A. 1993. Rapid proteolysis of ІкB- $\alpha$ is necessary for activation of transcription factor NF-кB. Nature 365: 182-185.

Hoffmann, A., Levchenko, A., Scott, M.L., and Baltimore, D. 2002. The ІкB-NF-кB signaling module: Temporal control and selective gene activation. Science 298: 11241-11245.

Hudson, B.P., Martinez-Yamout, M.A., Dyson, H.J., and Wright, P.E. 2000. Solution structure and acetyl-lysine binding activity of the GCN5 bromodomain. J. Mol. Biol. 304: 355-370.

Karin, M. and Lin, A. 2002. NF-кB at the crossroads of life and death. Nat. Immunol. 3: 221-227.

Kiernan, R., Bres, V., Ng, R.W., Coudart, M.P., El Messaoudi, S., Sardet, C., Jin, D.Y., Emiliani, S., and Benkirane, M. 2003. Post-activation turn-off of NF-кB-dependent transcription is regulated by acetylation of p65. J. Biol. Chem. 278: 27582766.

Lawrence, T., Bebien, M., Liu, G.Y., Nizet, V., and Karin, M. 2005. IKK $\alpha$ limits macrophage NF- $\mathrm{B}$ activation and contributes to the resolution of inflammation. Nature 434: 1138-1143.

Lewis, J., Burstein, E., Reffey, S.B., Bratton, S.B., Roberts, A.B., and Duckett, C.S. 2004. Uncoupling of the signaling and caspase-inhibitory properties of X-linked inhibitor of apoptosis. J. Biol. Chem. 279: 9023-9029.

Li, Q., Lu, Q., Hwang, J.Y., Buscher, D., Lee, K.F., IzpisuaBelmonte, J.C., and Verma, I.M. 1999a. IKK1-deficient mice exhibit abnormal development of skin and skeleton. Genes \& Dev. 13: 1322-1328.

Li, Q., Van Antwerp, D., Mercurio, F., Lee, K.F., and Verma, I.M. 1999b. Severe liver degeneration in mice lacking the IкB kinase 2 gene. Science 284: 321-325.

Linares, L.K., Kiernan, R., Triboulet, R., Chable-Bessia, C., Latreille, D., Cuvier, O., Lacroix, M., Le Cam, L., Coux, O., and Benkirane, M. 2007. Intrinsic ubiquitination activity of PCAF controls the stability of the oncoprotein Hdm2. Nat. Cell Biol. 9: 331-338.

Lois, C., Hong, E.J., Pease, S., Brown, E.J., and Baltimore, D. 2002. Germline transmission and tissue-specific expression 
of transgenes delivered by lentiviral vectors. Science 295: 868-872.

Maine, G.N., Mao, X., Komarck, C.M., and Burstein, E. 2007. COMMD1 promotes the ubiquitination of NF- $\mathrm{KB}$ subunits through a Cullin-containing ubiquitin ligase. $E M B O ~ J .26$ : 436-447.

Mattioli, I., Geng, H., Sebald, A., Hodel, M., Bucher, C., Kracht, M., and Schmitz, M.L. 2006. Inducible phosphorylation of NF- $\mathrm{BB}$ p 65 at serine 468 by T cell costimulation is mediated by IKKe. J. Biol. Chem. 281: 6175-6183.

Petroski, M.D. and Deshaies, R.J. 2005. Review: Funtion and regulation of cullin-ring ubiquitin ligases. Nat. Rev. Mol. Cell Biol. 6: 9-20.

Ramsey, C.S., Yeung, F., Stoddard, P.B., Li, D., Creutz, C.E., and Mayo, M.W. 2008. Copine-I represses NF-кB transcription by endoproteolysis of p65. Oncogene 27: 3516-3526.

Ryo, A., Suizu, F., Yoshida, Y., Perrem, K., Liou, Y.C., Wulf, G., Rottapel, R., Yamaoka, S., and Lu, K.P. 2003. Regulation of NF-кB signaling by Pin1-dependent prolyl isomerization and ubiquitin-mediated proteolysis of p65/RelA. Mol. Cell 12: 1413-1426.

Saccani, S., Marazzi, I., Beg, A.A., and Natoli, G. 2004. Degradation of promoter-bound p65/RelA is essential for the prompt termination of the nuclear factor $\mathrm{\kappa B}$ response. $I$. Exp. Med. 200: 107-113.

Sakurai, H., Chiba, H., Miyoshi, H., Sugita, T., and Toriumi, W. 1999. ІкB kinases phosphorylate NF-кB p65 subunit on serine 536 in the transactivation domain. J. Biol. Chem. 274: 30353-30356.

Schmitz, M.L. and Baeuerle, P.A. 1991. The p65 subunit is responsible for the strong transcription activating potential of NF-kB. EMBO I. 10: 3805-3817.

Schwabe, R.F. and Sakurai, H. 2005. IKK $\beta$ phosphorylates p65 at S468 in transactivaton domain 2. FASEB J. 19: 1758-1760.

Silverman, N. and Maniatis, T. 2001. NF-кB signaling pathways in mammalian and insect innate immunity. Genes \& Dev. 15: 2321-2342.

Tagwerker, C., Flick, K., Cui, M., Guerrero, C., Dou, Y., Auer, B., Baldi, P., Huang, L., and Kaiser, P. 2006. A tandem-affinity tag for two-step purification under fully denaturing conditions: Application in ubiquitin profiling and protein complex identification combined with in vivo cross-linking. Mol. Cell. Proteomics 5: 366-378.

Tanaka, T., Grusby, M.J., and Kaisho, T. 2007. PDLIM2-mediated termination of transcription factor NF- $\mathrm{kB}$ activation by intranuclear sequestration and degradation of the p65 subunit. Nat. Immunol. 8: 584-591.

Tanner, K.G., Trievel, R.C., Kuo, M.H., Howard, R.M., Berger, S.L., Allis, C.D., Marmorstein, R., and Denu, J.M. 1999. Catalytic mechanism and function of invariant glutamic acid 173 from the histone acetyltransferase GCN5 transcriptional coactivator. J. Biol. Chem. 274: 18157-18160.

Thomas, M.C. and Chiang, C.M. 2006. The general transcription machinery and general cofactors. Crit. Rev. Biochem. Mol. Biol. 41: 105-178.

Zhong, H., Voll, R.E., and Ghosh, S. 1998. Phosphorylation of NF-к B p65 by PKA stimulates transcriptional activity by promoting a novel bivalent interaction with the coactivator CBP/p300. Mol. Cell 1: 661-671. 


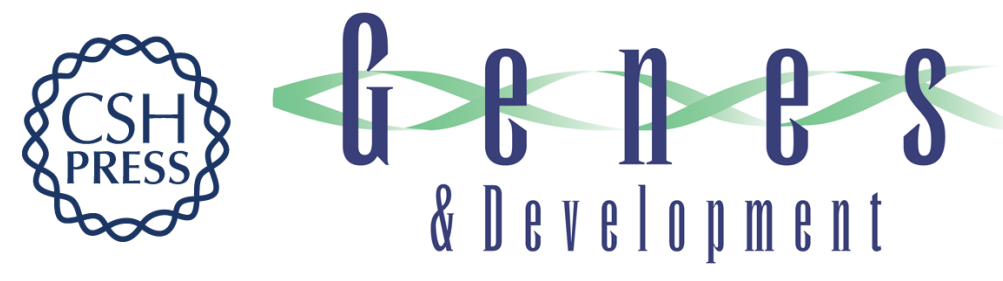

\section{GCN5 is a required cofactor for a ubiquitin ligase that targets NF- $\kappa$ B/RelA}

Xicheng Mao, Nathan Gluck, Duo Li, et al.

Genes Dev. 2009, 23:

Access the most recent version at doi:10.1101/gad.1748409

\section{Supplemental http://genesdev.cshlp.org/content/suppl/2009/04/02/23.7.849.DC1 Material}

References This article cites 38 articles, 21 of which can be accessed free at: http://genesdev.cshlp.org/content/23/7/849.full.html\#ref-list-1

\section{License}

Email Alerting

Receive free email alerts when new articles cite this article - sign up in the box at the top Service 